\title{
Enhanced in vivo visualization of the microcirculation by topical application of fructose solution confirmed with correlation mapping optical coherence tomography
}

Joey Enfield

James McGrath

Susan M. Daly

Martin Leahy 


\title{
Enhanced in vivo visualization of the microcirculation by topical application of fructose solution confirmed with correlation mapping optical coherence tomography
}

\author{
Joey Enfield, ${ }^{a}$ James McGrath, ${ }^{a}$ Susan M. Daly, ${ }^{a}$ and Martin Leahy ${ }^{a, b, \star}$ \\ ${ }^{a}$ Tissue Optics and Microcirculation Imaging Facility, National Biophotonics and Imaging Platform, Department of Physics and Energy, Ireland \\ ${ }^{b}$ National University of Ireland, Tissue Optics and Microcirculation Imaging Group, National Biophotonics and Imaging Platform, School of Physics, \\ Galway, Ireland
}

\begin{abstract}
Changes within the microcirculation can provide an early indication of the onset of a plethora of ailments. Various techniques have thus been developed that enable the study of microcirculatory irregularities. Correlation mapping optical coherence tomography (cmOCT) is a recently proposed technique, which enables mapping of vasculature networks at the capillary level in a noninvasive and noncontact manner. This technique is an extension of conventional optical coherence tomography (OCT) and is therefore likewise limited in the penetration depth of ballistic photons in biological media. Optical clearing has previously been demonstrated to enhance the penetration depth and the imaging capabilities of OCT. In order to enhance the achievable maximum imaging depth, we propose the use of optical clearing in conjunction with the cmOCT technique. We demonstrate in vivo a $13 \%$ increase in OCT penetration depth by topical application of a high-concentration fructose solution, thereby enabling the visualization of vessel features at deeper depths within the tissue. $\odot 2016$ Society of Photo-Optical Instrumentation Engineers (SPIE) [DOI: 10.1117/1.JBO.21.8.081212]
\end{abstract}

Keywords: optical clearing; optical coherence tomography; microcirculation imaging; correlation mapping; biomedical optical imaging. Paper 160053SSR received Jan. 28, 2016; accepted for publication May 2, 2016; published online Jun. 17, 2016.

\section{Introduction}

The existence and prevalence of various diseases ${ }^{1-5}$ induce changes within the microcirculation and resultantly may provide an early indication of the onset of disease. ${ }^{6}$ This has resulted in the development of various techniques, which enable the study of microcirculatory dynamics, ${ }^{7-10}$ borne out of recognition of the vitally important role that blood flow plays in the health of the individual. In recent years, there has been increased interest in functional extensions of the optical coherence tomography (OCT) technique, providing quantitative metrics of dynamic processes within the vasculature. These extensions include Doppler OCT, ${ }^{6}$ speckle variance OCT, ${ }^{11}$ and optical microangiography. ${ }^{12}$ Recently, our group has developed a tool for visualization of the microcirculation that exhibits no angular dependence and is highly sensitive to low-flow velocities (verified at detecting Brownian motion)_correlation mapping optical coherence tomography (cmOCT). ${ }^{13,14}$ The technique is an extension of conventional OCT, providing maps of the microcirculation structure through noncontact and noninvasive means. "Correlation mapping" is a processing step applied to OCT intensity data, isolating flow locations within the specimen in question.

The penetration depth of light into the tissue is a primary limiting factor in optical techniques and is similarly disadvantageous with regards to the cmOCT method. Scattering within

*Address all correspondence to: Martin Leahy, E-mail: martin.leahy @ nuigalway ie the tissue may be controlled and reduced through the administration of biocompatible chemical agents; this is termed optical clearing. Physically, the application of such products yields an increased penetration depth of light. ${ }^{12-15}$ To date, optical clearing has been achieved using various agents including glycerol, glucose, dimethyl sulfoxide, polypropylene glycol, polyethylene glycol, fructose, sorbitol, dextrose, and sucrose. ${ }^{15-20}$ In addition to enhancements in the achievable penetration depth, optical clearing methods have previously been demonstrated to yield increased contrast in OCT images. ${ }^{21}$ Enhanced OCT imaging has been demonstrated in a number of studies for various tissues including skin, stomach, esophagus, and sclera. ${ }^{21-25}$

The exact underlying physical mechanism of the optical clearing process remains an open discussion point. The combination of refractive index matching and skin dehydration due to the hyperosmotic nature of optical clearing agents (OCAs) is the most commonly accepted hypothesis. ${ }^{18}$ This would suggest that optical clearing should be related to the refractive index and the osmolarity of an agent; however, Choi et al. ${ }^{26}$ demonstrated no apparent correlation. Recent work by Hirshburg et al. ${ }^{15,17,27}$ has demonstrated that sugar alcohols such as glycerol can destabilize collagen structure, and the degree of collagen destabilization observed ex vivo corresponds with measured efficacy of OCAs.

Disruption of the stratum corneum (SC) using mechanical or chemical means may expedite the rate of OCA diffusion. The simplest method to achieve this is via intradermal needle

1083-3668/2016/\$25.00 @ 2016 SPIE 
injection $^{18,19}$ or through the use of a pneumatic jet device. ${ }^{28}$ In these methods, the OCA is placed in large concentrations beneath the $\mathrm{SC}$, diffuses throughout the surrounding tissue, and induces optical clearing. In addition to the disadvantageous invasive aspect of these methods, the introduction of high-concentration injections of OCAs, such as glycerol, has been shown to induce tissue necrosis, ${ }^{29,30}$ thus, limiting the suitability of glycerol as an in vivo OCA. Disruption of the SC may cover a large region (using, e.g., tape stripping ${ }^{31,32}$ or the application of a light mechanical abrasion ${ }^{33}$ ) or may be more localized to create a series of channels through the SC (using microneedles ${ }^{34}$ or thermal ablation of the $\mathrm{SC}^{35-37}$ ). These methods may result in tissue damage and unwanted after effects such as scabbing and erythema. All these methods (excluding injections) require a reservoir to maintain contact between the agent and the skin, which can limit the clinical applicability of the agents.

As a majority of reported OCAs are hydrophilic in nature, this limits their rate of diffusion across the SC layer of the skin. This can be enhanced using a carrier lipophilic molecule, such as azone, ${ }^{38}$ oleic acid, ${ }^{39}$ and thiazone. ${ }^{40}$ It has been demonstrated that fructose offers a greater reduction in scattering coefficient for ex vivo rodent skin, when lower molarities were present in the tissue than other agents tested (e.g., glycerol). ${ }^{17}$ The importance of this may be seen from the perspective that due to the limited diffusion rate across the skin, a lower fructose concentration can still produce an optical clearing effect. Due to their high water solubility, fructose gels can be produced in high concentrations when dissolved in water. ${ }^{41}$ The result is a high viscosity gel, which adheres to the tissue surface upon application. This removes the requirement for a reservoir to maintain the agent in contact with the tissue. The use of a highly concentrated solution also offers the benefit of enhancing the flux of the agents across the tissue due to the high-concentration gradient. Enhancing the flux of the agent into the tissue is a key issue for a practical topical OCA due to the barrier functions (i.e., SC) of the skin.
The cmOCT technique was originally demonstrated in small animal models by mapping mouse cerebral microcirculation. ${ }^{13}$ Recently, we have demonstrated the suitability of the technique for mapping the microcirculation structure for in vivo human skin at capillary level. ${ }^{14}$ The use of OCAs for imaging dermal blood flow through intact rat skin using laser speckle contrast imaging $^{42}$ has been previously reported. In this work, we propose the use of high-concentration fructose solution for in vivo dermal topical optical clearing. This will serve as a means of visualization enhancement for the cmOCT technique, to provide three-dimensional (3-D) blood flow information of deeper vascular features within the microcirculation.

\section{Methodology}

The high-concentration fructose gel utilized in this work was prepared by dissolving $10 \mathrm{~g}$ of fructose (Sigma-Aldridge: F9048) in $1.5 \mathrm{ml}$ of deionized water. This was achieved using a magnetic stirrer and heater (CAT: M6.1) and slowly adding the fructose into the water until fully dissolved. The solution was then allowed to cool to room temperature before application.

The cmOCT algorithm is utilized as a processing step on OCT intensity data. In this work, an unmodified commercial OCT system (OCM1300SS, Thorlabs Inc., Newton, New Jersey) has been utilized for imaging. The system is an Fourier domainOCT system using a swept source laser (SL1325-P16, Thorlabs Inc.). The laser has a center wavelength of $1325 \mathrm{~nm}$ and operates at a scanning rate of $16 \mathrm{kHz}$ providing an axial resolution of $\sim 12 \mu \mathrm{m}$. The sample arm is focused with an LSM03 (Thorlabs Inc.) scanning lens, which provided a lateral resolution of $25 \mu \mathrm{m}$ and $X-Z$ imaging range up to $11 \times 11 \mathrm{~mm}$. The system is capable of acquiring and saving 3-D OCT volumes consisting of $1024 \times 1024 \mathrm{~A}$-scans in $\sim 70 \mathrm{~s}$. The cmOCT algorithm identifies flow using the decorrelation between adjacent $\mathrm{B}$-scans. This is calculated by cross correlating between a grid from frame $\mathrm{A}\left(I_{\mathrm{A}}\right)$ to the same grid from frame $\mathrm{B}\left(I_{\mathrm{B}}\right)$ using the below equation

$\operatorname{cmOCT}(x, y)=\sum_{p=0}^{M} \sum_{q=0}^{N} \frac{\left[I_{\mathrm{A}}(x+p, y+q)-\overline{I_{\mathrm{A}}(x, y)}\right]\left[\left[I_{\mathrm{B}}(x+p, y+q)-\overline{I_{\mathrm{B}}(x, y)}\right]\right]}{\sqrt{\left[I_{\mathrm{A}}(x+p, y+q)-\overline{I_{\mathrm{A}}(x, y)}\right]^{2}\left[I_{\mathrm{B}}(x+p, y+q)-\overline{I_{\mathrm{B}}(x, y)}\right]^{2}}}$,

where $M$ and $N$ define the correlation grid size and $\bar{I}$ is the mean value of this grid. The defined grid is then mapped across the full $X-Y$ image resulting in a two-dimensional correlation map. All data presented in this paper have been processed using a kernel size of $7 \times 7$. There are a number of factors that influence the appropriate choice of kernel size, including correlation signal-to-noise ratio (CSNR), frame rate, processing time, and angiogram resolution. With respect to CSNR values, these will increase for the images as kernel size increases. This is expected, as larger kernels will not be as susceptible to slight changes in structure in the OCT image as smaller kernels. Larger kernels are also more resilient to noise in the image. However, there is a number of significant drawbacks to using large kernels. First of all, they require much more processing time. Second, larger kernels will cause blurring in the correlation image and will make smaller regions of flow undetectable. From an in vivo perspective, if the aim is to detect smaller capillaries or to measure capillary diameter this will be a significant issue. Thus, the optimal relationship between spatial resolution and CSNR should be found for each specific experimental task.

In Table 1, we present a study of some of these factors. As can be seen, as we increase kernel size, the angiogram CSNR increases. However, the processing time increases and the resolution of the angiogram decreases. The aim is to find the optimum kernel size for a given scenario, giving acceptable resolution, CSNR, and a reasonable processing time.

The above CSNR and processing times were calculated from $928 \times 928 \times 512$ pixels volume OCT images of a capillary tube phantom with no image registration being performed.

Another factor that must be taken into account is the background noise, which has low correlation and thus must be suppressed in the cmOCT processing. This is achieved through masking the correlation image with a structural mask. This 
Table 1 The impact of kernel size on CSNR and processing time. In this study, we felt $7 \times 7$ was a good compromise.

\begin{tabular}{lcc} 
Kernel size & CSNR & Processing time (s) \\
\hline $3 \times 3$ & 0.89 & 40 \\
$5 \times 5$ & 2.80 & 47 \\
$7 \times 7$ & 5.13 & 65 \\
$9 \times 9$ & 8.23 & 97 \\
$11 \times 11$ & 12.9 & 127 \\
$13 \times 13$ & 14.0 & 168 \\
$15 \times 15$ & 15.5 & 221 \\
\hline
\end{tabular}

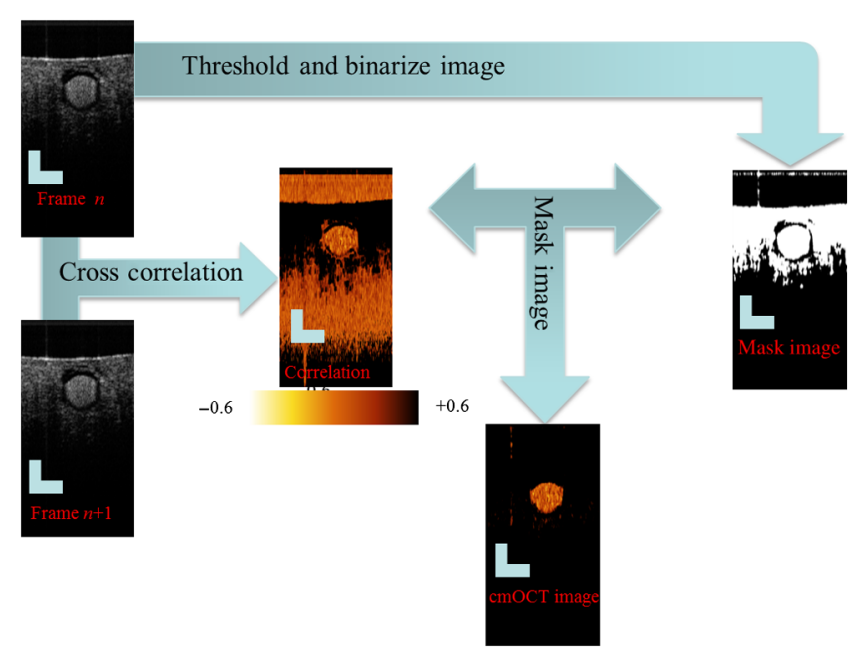

Fig. 1 Processing steps for the cmOCT algorithm. Higher correlation is shown as darker color while lower correlation is shown as brighter color. The figure shows a 300- $\mu$ m capillary tube containing Intralipid ${ }^{\mathrm{TM}}$ solution moving under Brownian motion embedded in excised porcine tissue. Scale bars are $300 \mu \mathrm{m}$. mask is generated by performing a kernel blur, followed by a binary threshold on the source OCT structural image. The selection of a threshold value is chosen; generally, this value corresponds to known structurally static regions within the processed image. The choice of the mean pixel value of these static regions is termed the mean background value and only (decorrelated) regions of flow remain upon subtraction of this term. This binary mask is then applied to the correlation map and a cmOCT image is generated. The resulting cmOCT image contains correlation values in the range 0 to \pm 1 , i.e., weak correlation and strong correlation, respectively. This allows for immediate discernment of flow regions as higher correlation represents static regions of the sample and low correlation represents regions of flow within the sample. In this work, static features are empirically chosen and have a correlation value $>0.6$; thus, color mapping is applied below this range. These processing steps are shown in Fig. 1.

\section{Results}

\subsection{Phantom Imaging}

A phantom was fabricated to demonstrate the enhanced visualization of flow at deeper depths achievable through the application of optical clearing using cmOCT. The phantom consisted of a section of ex vivo blood-free porcine skin, obtained from a local abattoir. A 300- $\mu$ m-diameter capillary tube was embedded at a shallow angle to enable visualization of flow through various depths within the tissue. A 3\% Intralipid solution was placed within the tube and allowed to move under Brownian motion. Prior to clearing, the phantom was imaged using the OCT system. For clearing, the phantom was suspended in a holder and immersed in the OCA so that the phantom could be penetrated from all sides; although not representative of in vivo testing, this was done preliminarily in order to achieve the best level of clearing for the in vitro phantom. The sample was then immersed in a 10-M fructose solution and imaged immediately after fructose application and then at 15 min intervals; the effect of this immersion is shown in Fig. 2. As the OCA enters the tissue, there is a resulting reduction in the scattering within the tissue. This enhances the capability of the impinging light to
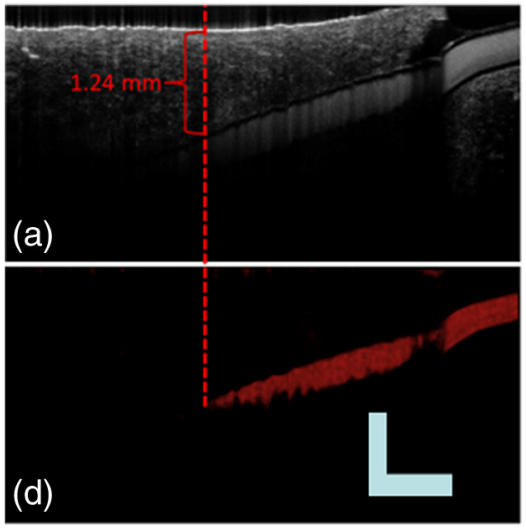

$+0.6$
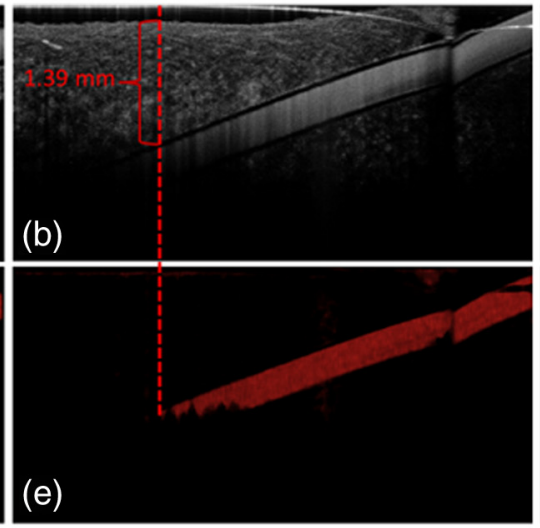

(e)

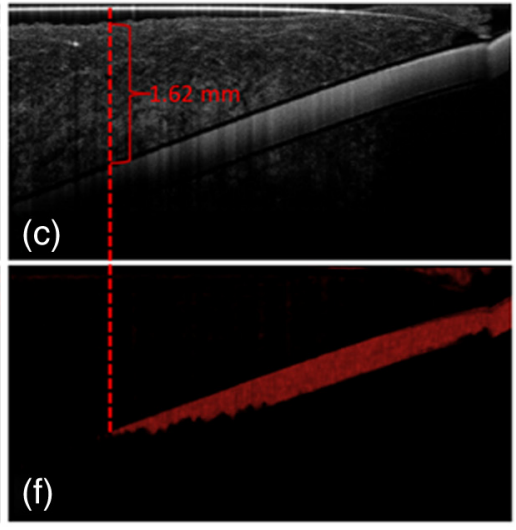

$-0.6$

Fig. 2 Enhanced visualization of depth using cmOCT. (a-c) The structural OCT image at time intervals of 0,15 , and 30 min after application of OCA. (d-f) The resulting detected $\mathrm{CmOCT}$ signal at similar times. Scale bars are $1 \mathrm{~mm}$. 
probe further into tissue yielding an increased OCT signal with depth and thus enables cmOCT (and indeed standard OCT) to detect deeper flow signatures. To illustrate this enhanced visualization of flow at deeper depths, the maximum depth under the tissue that flow was detected was estimated using cmOCT. The maximum depth was taken as the deepest point, where correlation values of $<0.6$ are obtained. Prior to immersion in the OCA [Figs. 2(a) and 2(d)], the maximum depth at which flow was detected using cmOCT was $\sim 1.24 \mathrm{~mm}$. Following immersion of the tissue phantom into the OCA, maximum imaging depth was seen to increase to $1.39 \mathrm{~mm}$ after 15 min [Figs. 2(b) and 2(e)] and following $30 \mathrm{~min}$ of immersion increased to $1.62 \mathrm{~mm}$ [Figs. 2(c) and 2(f)]. The results clearly illustrate that using fructose as an OCA can greatly enhance the maximum depth from which a cmOCT signal can be obtained.

\subsection{In Vivo Imaging}

To assess the suitability of the OCA at enhancing the visualization of deeper vessels in vivo, capillaroscopy imaging was performed. The capillaroscopy imaging was implemented using a USB microscope (N87fx, Maplin Ltd., 200× magnification) and a digital camera (Canon G9).

The in vivo work was performed on the volar forearm region of a healthy male volunteer aged 25 with no history of skin conditions. The study was approved by the ethics commission of the University of Limerick and full written consent was obtained from the subject. Prior to commencement of the study, the forearm of the subject was cleaned using lukewarm water and dried. The subject was then placed in an upright sitting position and allowed $10 \mathrm{~min}$ to acclimatize to the laboratory environment. After the acclimatization period, the region under investigation was imaged to assess the initial visibility of the microcirculation before the application of the OCA. The imaging protocol used was to first perform a full-field digital camera image of the forearm region followed by several capillaroscopy images at magnifications of $100 \times$ and $200 \times$. The OCA utilized in this work consisted of the high-concentration fructose gel that was produced using the aforementioned technique. The gel was applied to the skin and massaged for $\sim 10 \mathrm{~s}$ to form a smooth uniform thick layer. To assess the changes in the visibility of the microcirculation, the region was imaged immediately upon application of the OCA and then at 20 min intervals. The results of this are shown in Fig. 3.

The figure illustrates the enhanced visualization of the microcirculation that is achieved using the topical fructose gel. The results demonstrate that prior to the application of the OCA, the underlying microcirculation could not be clearly visualized [see Fig. 3, before clearing $(\mathrm{a}-\mathrm{c})$ ]. Initially after the topical application, there is a minor change in visualization of the

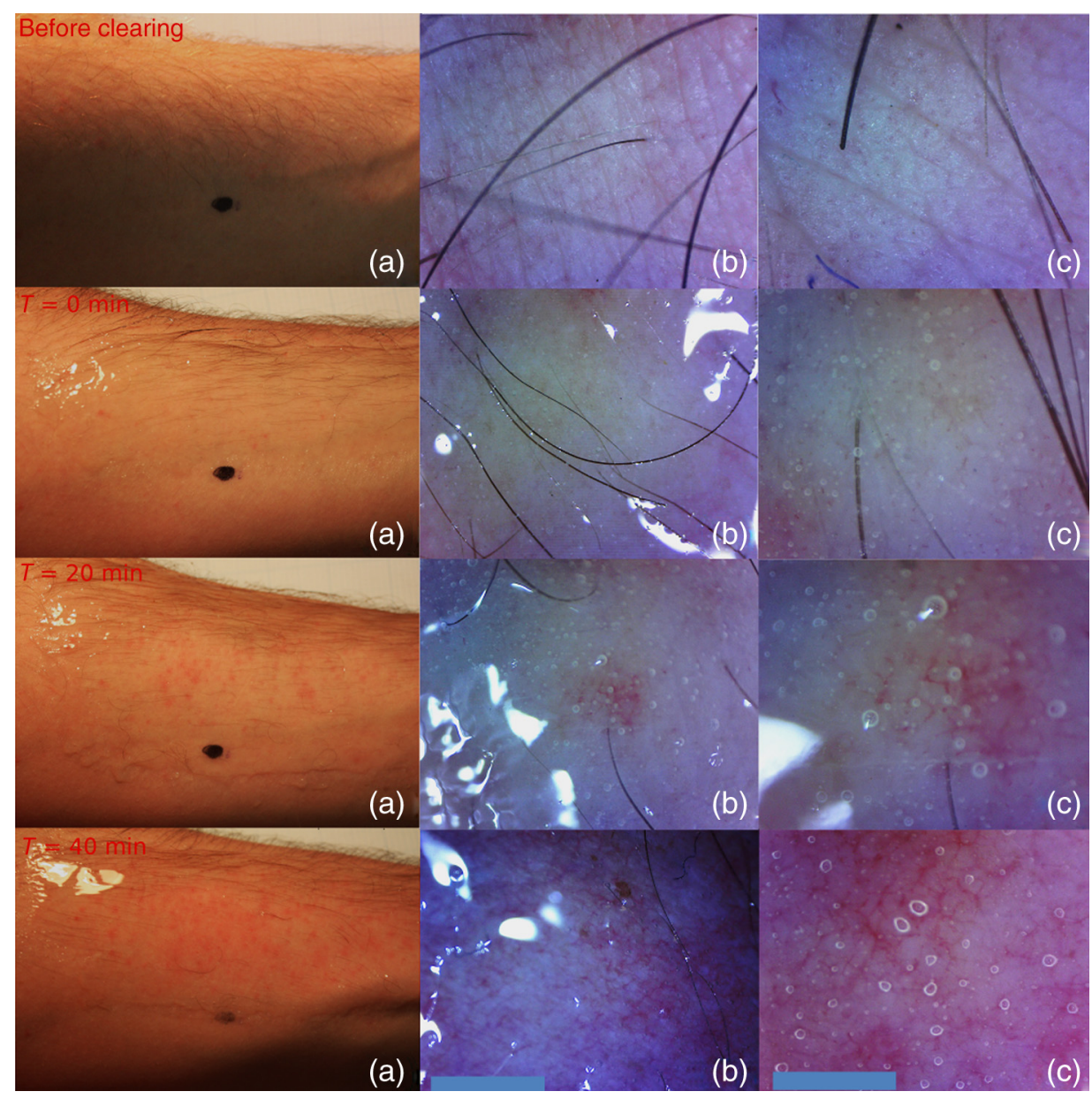

Fig. 3 Enhanced visualization of the microcirculation for various times after topical application of the OCA. (a) Digital RGB imaging of the cleared region (2-cm scale bar), (b) $100 \times$ magnification of region marked in a (2-mm scale bar), and (c) $200 \times$ magnification of region marked in (a) (400- $\mu \mathrm{m}$ scale bar). 
microcirculation (see Fig. 3, $T=0 \mathrm{~min}$ ). The tips of the capillary loops can be visualized with enhanced clarity. However, the overall microcirculation structure is not clearly visible. The effects of the OCA became apparent within 20 min from initial topical application. Initially, the appearance of cleared vessels could be visibly seen forming at the base of certain hair follicles; this is clearly observable in Fig. 3(a), $T=20 \mathrm{~min}$. When these regions were examined under higher magnifications, an underlying vessel network could be clearly visualized surrounding the follicle; this is shown in Figs. 3(b) and 3(c), $T=20 \mathrm{~min}$. Due to the large molecular size of fructose and its hydrophilic nature, the penetration rate through the SC would be expected to be quite low. These results would indicate that the fructose gel uses the hair follicle as a penetration route to bypass the layer and diffuses throughout the surrounding tissue. This induces a reduction in the tissue scattering due to the optical clearing effect and forms a transparent window to the underlying microcirculation. The increased redness of the tissue would follow due to a larger portion of light interacting with the blood in the microcirculation of the skin. The OCA continues to enter the tissue through the hair follicles, which is aided by the constant contact of the fructose gel on the surface of the skin. The result of this is that the transparent windows grow outward from each hair follicle and merge to induce a large transparent region of the skin. This can be seen in Fig. 3, $T=40 \mathrm{~min}$, where a large vessel network can be clearly identified. Due to the complexity of the biological environment, each hair follicle may not have ready access to the underlying vessel network for OCA diffusion; therefore, the network surrounding each hair shaft base may not be cleared. Irrespective of this, the extent of the transparent window is clearly evident. During the experiment, no physiological changes to the skin occurred other than those induced by the osmotic effect of the OCA. The tissue site was monitored for several days after the experiment and no damage or reaction of the tissue was observed. Capillaroscopic imaging clearly demonstrates that the topical application of a fructose solution results in enhanced visualization of the underlying microcirculation. This would suggest that cmOCT could obtain additional information of vessel structures from deeper into the tissue.

To assess the effect of the OCA on the penetration depth and enhanced cmOCT imaging, testing was performed in vivo on the volar forearm. A similar procedure was followed as used during the capillaroscopy imaging. A 3-D OCT scan of the forearm was acquired over an area of $3 \times 3 \mathrm{~mm}$ with using $1024 \times 1024$ A-scans. The OCA was then applied and left in contact for $40 \mathrm{~min}$. After this, time imaging was repeated at approximately the same location. The resulting data sets were processed using the cmOCT processing technique using a $7 \times 7 \mathrm{kernel}$; the results of this are shown in Fig. 4. This result clearly illustrates that a higher density of vessels is visualized in the maximum intensity projection (MIP) image after the optical clearing of the tissue was performed. Due to the inherent difficulty in repositioning the sample to the exact prior location, the figures have a slight offset; however, for ease of illustration, several arrows have been included in Fig. 4 to mark the same key features present in both instances, before and after agent application.

In order to assess the enhanced depth visualization and the location of the detected vessels, the change in the reflected OCT signal for the fully scanned volume was computed. Due to the natural curvature and orientation of the skin, it was not sufficient to directly average each adjacent A-scan. The surface of the skin (a)

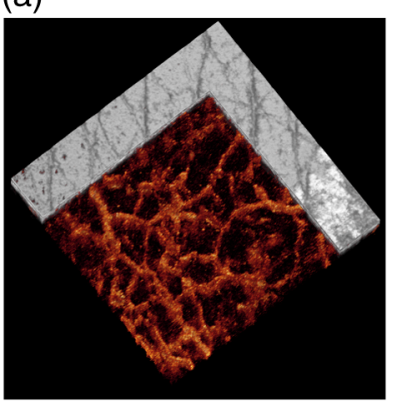

(b)

(c)
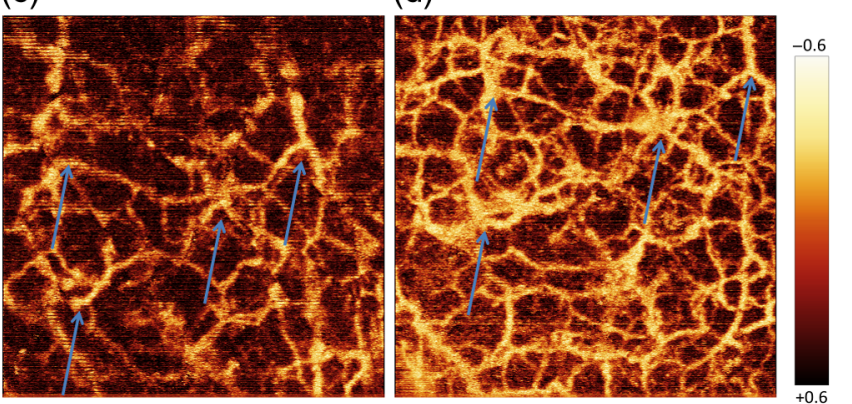

Fig. 4 Effects of optical clearing on detected cmOCT vascular structure for the volar forearm. 3-D render of structural and cmOCT volume with accompanying movies. (a) Before clearing (Video 1, QuickTime, 1.71 MB) [URL: http://dx.doi.org/10.1117/1.JBO.21.8.081212.1]. and (b) after clearing (Video 2, QuickTime, 1.81 MB) [URL: http://dx.doi. org/10.1117/1.JBO.21.8.081212.2]. MIP of cmOCT of the volar forearm with arrows marks identifying key features (a) before clearing and (b) after clearing. Region shown is $3 \times 3 \mathrm{~mm}$.

was first estimated. All A-scans were then aligned based on the surface position and the average of all $1024 \times 1024$ A-scans was calculated. The estimated surface was also used to determine normalized cmOCT vessel density as a function of depth. The vessel density parameter is calculated as the fractional area of detected vessels. This provides a method of comparison between the detected vessels before and after clearing. Figure 5 shows the change in the reflected structural OCT signal and the cmOCT vessel density as a result of the optical clearing process. Examination of the structural OCT signal [Fig. 5(a)] and the ratio of OCT signal [Fig. 5(b)] shows that at deeper depths $(>300 \mu \mathrm{m})$ an increase in the detected signal is apparent (a drop in the surface reflections after OCA application may be explained by the increased light coupling into the specimen). This is due to a reduction in the scattering coefficient of the tissue layers due to the optical clearing effect. To assess this enhanced penetration depth achieved using the OCA, the change in the scattering coefficient $\left(\mu_{\mathrm{s}}\right)$ can be estimated by fitting the OCT signal using a single scattering model ${ }^{43}$

$I(z) \propto \sqrt{\exp \left(-2 \mu_{\mathrm{S}} z\right)}$.

The marked region between $X$ and $Y$ in Fig. 5(a) was fitted, both before and after clearing, using Eq. (2) and the effective scattering coefficients estimated. Although the diffusion of fructose induces local refractive index changes, variations in optical thickness as detected by OCT may result. However, as experimental conditions were consistent with time, the increased intensity in OCT signal from deeper depths may be supported by the enhanced visualization of vessels in response to the 

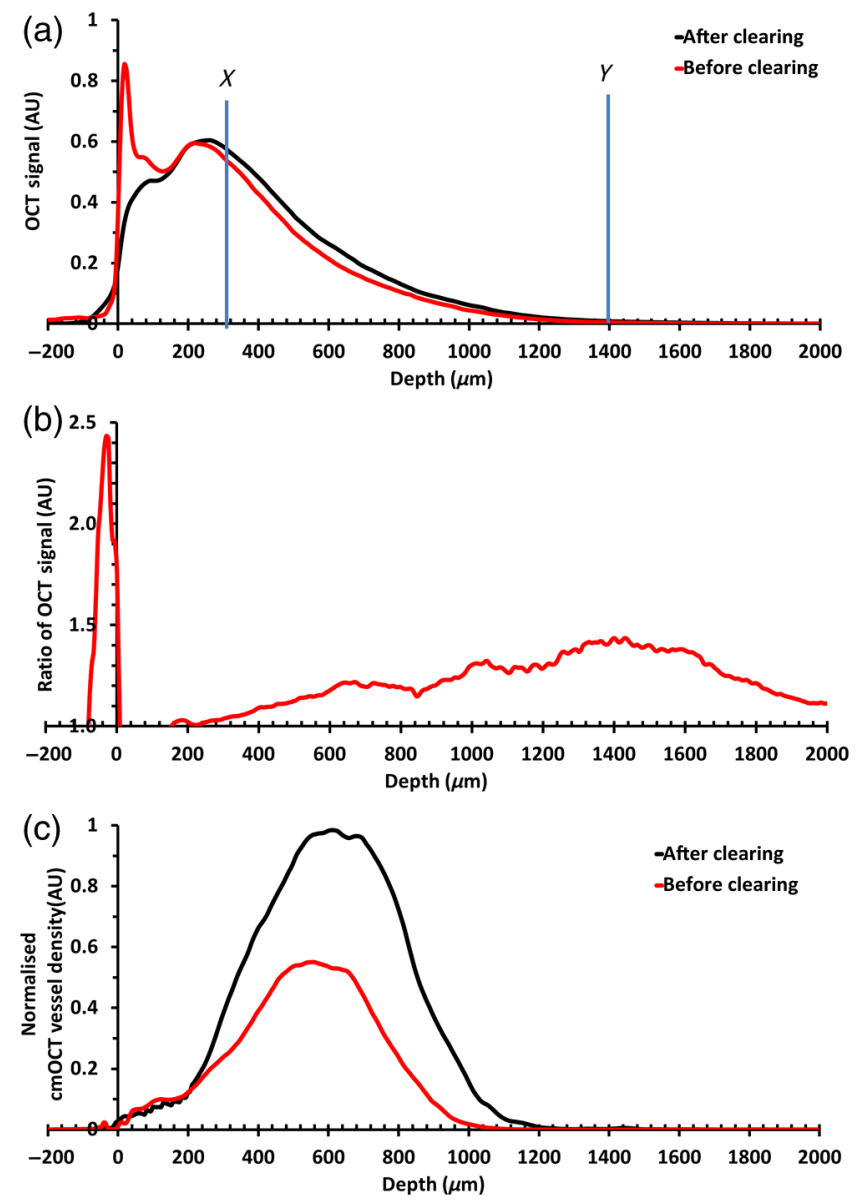

Fig. 5 (a) Mean OCT signal, (b) ratio of before and after clearing, and (c) normalized cmOCT vessel density parameter. The marked region between $X$ and $Y$ in (a) was fitted, both before and after clearing, using Eq. (2) and the effective scattering coefficients estimated.

clearing effect. Prior to application of the OCA the scattering coefficient $\left(\mu_{\mathrm{S}}\right)$ has been estimated as $9.4 \pm 0.31 \mathrm{~mm}^{-1}$; after optical clearing $\mu_{\mathrm{S}}$ decreases to $8.29 \pm 0.23 \mathrm{~mm}^{-1}$. This reduction in scattering coefficient would indicate an increase of the $1 / \mathrm{e}$ penetration by $13 \%$. This clearly illustrates that the OCA has induced a reduction in the scattering within the tissue and thus enhanced the penetration depth of the OCT signal. When the cmOCT vessel density is analyzed, it is clear that there is a marked change in the detected signal after the application of the OCA [Fig. 5(c)]; 2 $\times$ increase in the magnitude of the vessel density is detected after clearing. This indicates a tissue response to the OCA by increasing blood flow to the region, which would indicate a possible vessel dilation or erythema. This result illustrates the suitability of cmOCT to study the effects of OCAs on the microcirculation. It is also clear, however, that there is an increase in the vessel density detected at deeper depths ( $>1000 \mu \mathrm{m})$ after application of the OCA. This clearly illustrates that optical clearing can enhance the cmOCT imaging depths within the tissue. To better illustrate this point, the MIP can be color coded as a function of depth. Figure 6 shows the data from Fig. 4, which has been manually aligned and color mapped based on the depth of the detected vessel. In the figure, warmer hues represent the location of deeper vessels. Yellow arrows are used to point to the new deeper vessels and their color shows their depth. In addition to increased vessel (a)

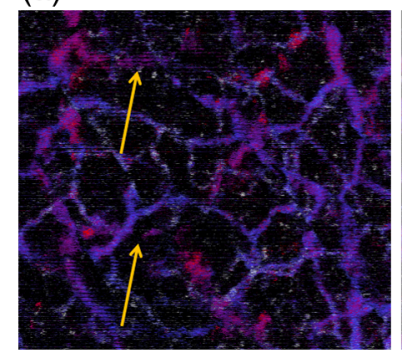

(b)

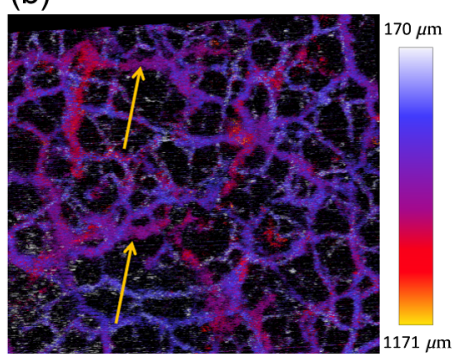

Fig. 6 Depth color coded cmOCT detected vessels (from Fig. 4), which are manually aligned, (a) before and (b) after clearing. Yellow arrows are used to point the new deeper vessels and their color shows their depth. Region shown is $3 \times 3 \mathrm{~mm}$.

density, several deeper vessels are evident after the OCA application. Overall, these results illustrate that optical clearing can enhance the achievable depth and ergo visualization of vessel flow using the cmOCT method.

\section{Summary}

In this paper, we have demonstrated that a topical application of a high-concentration fructose solution provides optical clearing and enhanced visualization of the vasculature at deeper depths used in conjunction with cmOCT imaging. Due to the large molecular size and hydrophilic nature of fructose, this was unexpected prior to experimentation. A tentative explanation for the fructose diffusion mechanism may be proffered by the bypassing of the SC by the OCA through the hair follicles and subsequently diffusing through the surrounding tissue. Although the relatively low hair follicle density in the volar forearm region may lend credence to this hypothesis, repetition of this work on in vivo regions of varying follicle density will be required to verify this claim. In parallel with this study, Wang et al. ${ }^{44}$ have provided evidence of a molecular basis (hydrogen bonds) for the finding that fructose is better in vivo.

The application of this fructose solution on a tissue phantom resulted in a $30 \%$ increase in the achievable maximum cmOCT imaging depth. In vivo application of the OCA resulted in a $13 \%$ increase in the detectable OCT signal. The enhanced penetration depth enabled the detection of vessels at greater depths within the tissue, which were previously not detected prior to application of the OCA. Overall, the results have clearly shown that by the topical application of fructose as an OCA, the maximum image depth achievable can be enhanced and harnessed by the cmOCT technique, to provide 3-D blood flow information of deeper vascular features within the microcirculation.

\section{Acknowledgments}

This research was supported by the National Biophotonics Imaging Platform Ireland funded under the Higher Education Authority PRTLI Cycle 4 and cofunded by the Irish Government and the European Union-Investing in your future. The authors would also like to acknowledge the support of Irish Research Council for Science, Engineering and Technology.

\section{References}

1. J. Folkman, "Tumor angiogenesis factor," Cancer Res. 34, 2109 (1974).

2. R. Bull, D. O. Bates, and P. S. Mortimer, "Intravital video-capillaroscopy for the study of the microcirculation in psoriasis," $\mathrm{Br} . J$. Dermatol. 126(5), 436-445 (1992). 
3. M. Cutolo, W. Grassi, and M. Matucci Cerinic, "Raynaud's phenomenon and the role of capillaroscopy," Arthritis Rheum. 48, 3023-3030 (2003).

4. E. Kohner, "Dynamic changes in the microcirculation of diabetics as related to diabetic microangiopathy," Acta Med. Scand. 197, 41-47 (1975).

5. M. Smith et al., "Whole blood viscosity and microvascular abnormalities in Alzheimer's disease," Clin. Hemorheol. Microcirc. 41, 229-239 (2009).

6. X. Wang, T. Milner, and J. Nelson, "Characterization of fluid flow velocity by optical Doppler tomography," Opt. Lett. 20, 1337-1339 (1995).

7. J. O'Doherty et al., "Sub-epidermal imaging by polarised light rejection for assessment of microvascular patency," Skin Res. Technol. 13(4), 472-484 (2007).

8. J. O'Doherty et al., "Use of tissue viability imaging (TiVi) in the assessment of divergent beam UVB phototesting," Arch. Dermatol. Res. 33(2), 78-79 (2010).

9. M. J. Leahy, Ed., Microcirculation Imaging, Wiley-VCH, Berlin, Germany (2011).

10. J. O'Doherty et al., "Comparison of instruments for investigation of microcirculatory blood flow and red blood cell concentration," $J$. Biomed. Opt. 14(3), 034025 (2009).

11. J. Barton and S. Stromski, "Flow measurement without phase information in optical coherence tomography images," Opt. Express 13, 52345239 (2005).

12. R. Wang et al., "Three dimensional optical angiography," Opt. Express 15, 4083-4097 (2007).

13. E. Jonathan, J. Enfield, and M. Leahy, "Correlation mapping method for generating microcirculation morphology from optical coherence tomography (OCT) intensity images," J. Biophotonics 4(9), 583-587 (2011).

14. J. Enfield, E. Jonathan, and M. J. Leahy, "In-vivo imaging of the microcirculation of the volar forearm using correlation mapping optical coherence tomography (cmOCT)," Biomed. Opt. Express 2(5), 1184-1193 (2011).

15. A. Yeh et al., "Reversible dissociation of collagen in tissues," J. Invest. Dermatol. 121, 1332-1335 (2003).

16. A. T. Yeh and J. Hirshburg, "Molecular interactions of exogenous chemical agents with collagen-implications for tissue optical clearing," J. Biomed. Opt. 11(1), 014003 (2006).

17. J. Hirshburg et al., "Correlation between collagen solubility and skin optical clearing using sugars," Lasers Surg. Med. 39(5) (2007).

18. G. Vargas et al., "Use of an agent to reduce scattering in skin," Lasers Surg. Med. 24, 133-141 (1999).

19. E. Galanzha et al., "Skin backreflectance and microvascular system," J. Phys. D Appl. Phys. 36, 1739-1746 (2003).

20. Z. Mao et al., "Influence of alcohols on the optical clearing effect of skin in vitro," J. Biomed. Opt. 13, 021104 (2008).

21. R. Wang et al., "Concurrent enhancement of imaging depth and contrast for optical coherence tomography by hyperosmotic agents," J. Opt. Soc. Am. B 18, 948-953 (2001).

22. Y. H. He and R. K. K. Wang, "Dynamic optical clearing effect of tissue impregnated with hyperosmotic agents and studied with optical coherence tomography," J. Biomed. Opt. 9, 200-206 (2004).

23. R. K. Wang and J. B. Elder, "Propylene glycol as a contrasting agent for optical coherence tomography to image gastrointestinal tissues," Lasers Surg. Med. 30, 201-208 (2002).

24. X. Guo et al., "In vivo quantification of propylene glycol, glucose and glycerol diffusion in human skin with optical coherence tomography," Laser Phys. 20, 1849-1855 (2010).

25. K. V. Larin et al., "Potential application of optical coherence tomography for noninvasive monitoring of glucose concentration," Proc. SPIE 4263, 83 (2001)

26. B. Choi et al., "Determination of chemical agent optical clearing potential using in vitro human skin," Lasers Surg. Med. 36(2), 72-75 (2005).

27. J. M. Hirshburg et al., "Molecular basis for optical clearing of collagenous tissues," J. Biomed. Opt. 15 (5), 055002 (2010).

28. C. M. Cilip et al., "Application of an optical clearing agent during noninvasive laser coagulation of the canine vas deferens," J. Biomed. Opt. 15(4), 048001 (2010)

29. J. M. Hirshburg et al., "Influence of glycerol with different concentrations on skin optical clearing and morphological changes in vivo," Proc. SPIE 7278, 72781T (2009).
30. R. McNichols et al., "Temporary dermal scatter reduction: quantitative assessment and implications for improved laser tattoo removal," Lasers Surg. Med. 36, 289-296 (2005).

31. S. Chernova et al., "Dynamics of optical clearing of human skin in vivo," Proc. SPIE 4162, 227 (2000).

32. H. Schaefer and T. Redelmeier, Skin Barrier: Principles of Percutaneous Absorption, S. Karger AG, Basel, Switzerland (1996).

33. O. Stumpp, B. Chen, and A. Welch, "Using sandpaper for noninvasive transepidermal optical skin clearing agent delivery," J. Biomed. Opt. 11, 041118 (2006).

34. J. G. Enfield et al., "In vivo dynamic characterization of microneedle skin penetration using optical coherence tomography (OCT)," J. Biomed. Opt. 15(4), 046001 (2010).

35. S. Lee et al., "Photomechanical delivery of 100-nm microspheres through the stratum corneum: implications for transdermal drug delivery," Lasers Surg. Med. 31, 207-210 (2002).

36. O. Stumpp et al., "Enhancement of transepidermal skin clearing agent delivery using a $980 \mathrm{~nm}$ diode laser," Lasers Surg. Med. 37, 278-285 (2005).

37. V. Tuchin et al., "Optical clearing of skin using flashlamp-induced enhancement of epidermal permeability," Lasers Surg. Med. 38, 824-836 (2006)

38. X. Xu and Q. Zhu, "Evaluation of skin optical clearing enhancement with Azone as a penetration enhancer," Opt. Commun. 279, 223-228 (2007).

39. J. Jiang and R. Wang, "The clinical availability of oleic acid as an enhancer in optical clearing of skin tissue in vitro," Proc. SPIE 5696, 193 (2005).

40. J. Jiang et al., "Availability of thiazone as an enhancer for optical clearing of skin tissue in vitro," Proc. SPIE 6858, 68580N (2008).

41. A. Flood et al., "Refractive index, viscosity, density, and solubility in the system fructose+ ethanol+ water at $30^{\circ} \mathrm{C}, 40^{\circ} \mathrm{C}$, and $50^{\circ} \mathrm{C}$," J. Chem. Eng. Data 41, 418-421 (1996).

42. D. Zhu et al., "Imaging dermal blood flow through the intact rat skin with an optical clearing method," J. Biomed. Opt. 15(2), 026008 (2010).

43. P. Lee, W. Gao, and X. Zhang, "Performance of single-scattering model versus multiple-scattering model in the determination of optical properties of biological tissue with optical coherence tomography," Appl. Opt. 49, 3538-3544 (2010).

44. J. Wang et al., "Sugar-induced skin optical clearing: from molecular dynamics simulation to experimental demonstration," IEEE J. Sel. Top. Quantum Electron. 20(2), 7101007 (2014).

Joey Enfield graduated with a PhD from the Physics Department at UL in 2011. He has previously completed his BSc in applied physics in 2006 with a first class honors degree. Upon graduation he was awarded an IRCSET scholarship to undertake a PhD program in biomedical optics. His work focused on the area of optical clearing and cmOCT.

James McGrath is a student at the National University of Ireland, Galway. He received his BSc and HDipAppSc degrees in applied physics and electronics and software design and development from the National University of Ireland, Galway, in 2010 and 2011, respectively. His current research interests include high-resolution OCT and microcirculation imaging. $\mathrm{He}$ is a member of SPIE.

Susan M. Daly (née McElligott) completed her BSc in applied physics graduating with a first class honors degree in 2009. The same year she was awarded an IRCSET scholarship to pursue her PhD studies in biophotonics. Her work pertains to the design, development, and application of noninvasive correlation-based imaging systems aimed towards in vivo diagnostic applications, utilizing both morphological (structure) and functional (circulation) information of the vasculature.

Martin Leahy is a serial entrepreneur having been technical and/or managerial lead of several successful spin-out companies in biophotonics and energy. His main research interest is in the advancement of existing technologies, such as laser Doppler and laser speckle as well as the development of new modalities, such as TiVi and cmOCT for 2-D, 3-D, and 4-D imaging of the microcirculation. In 2010 he delivered a dozen international invited lectures and published more than a dozen ISI journal articles. He was appointed chair of applied physics at NUI Galway in April 2011. 